\title{
Specialized Genetic Algorithm of Chu-Beasley Applied to the Distribution System Reconfiguration Problem Considering Several Demand Scenarios
}

\author{
Simone S. F. Souza, Ruben Romero, Senior Member IEEE \\ Electrical Engineering Department \\ UNESP - Univ. Estadual Paulista \\ Ilha Solteira, SP, Brazil \\ simonefrutuoso.mat@gmail.com, ruben@dee.feis.unesp.br
}

\author{
Jorge Pereira \\ INESC TEC and Faculty of \\ Economics, University of Porto \\ Porto, Portugal \\ jpereira@inesctec.pt
}

\author{
J. T. Saraiva \\ INESC TEC and Faculty of \\ Engineering, University of Porto \\ Porto, Portugal \\ jsaraiva@fe.up.pt
}

\begin{abstract}
This paper describes the application of the specialized genetic algorithm of Chu-Beasley to solve the Distribution System Reconfiguration, DSR, problem considering different demand scenarios. This algorithm is an approach inspired in the natural selection and evolution of species. The reconfiguration problem of distribution networks taking into account different demand scenarios aims at identifying the most adequate radial topology of a distribution system assuming that this topology is used for all demand scenarios under study. This search is driven by the minimization of the cost of energy losses in the network along a full operation year. The performance of the algorithm is evaluated considering test systems having 33, 70, 84 and 136 buses and a real system with 417 buses. The obtained results confirm the robustness and efficiency of the developed approach and its potential to be used in distribution control centers.
\end{abstract}

Index Terms-Distribution System Reconfiguration, Demand scenarios, Specialized Genetic Algorithm of Chu-Beasley, Mixed-Integer Nonlinear Programming Problem.

\section{INTRODUCTION}

The Distribution System Reconfiguration (DSR) problem aims at identifying the most adequate topology for a distribution system taking advantage of the opening or closure of breakers or sectionalizers, while maintaining the radial nature of the network and the voltage levels within the pre specified ranges. In most cases, the DSR problem is solved minimizing the active losses in the network but other criteria can also be considered as the improvement of the voltage profile, the reliability of the network, the capacity to isolate faults or the definition of plans to do preventive maintenance actions [1].

The DSR problem has a combinatorial nature and it can be modeled as a mixed-integer nonlinear optimization problem (MINLP) [2], driven by the minimization of the active power losses in the network, subjected to a number of constraints related with the operation of the system. These constraints are typically related with the radial nature of the network, the nodal voltage limits, the branch current limits while enforcing the first and the second Kirchhoff Laws. The majority of the approaches to this problem available in the literature just consider one demand scenario taken as fixed as the ones in [2
- 7], which means that the identified topology is adequate just for the demand that was analyzed. However, some authors consider a number of demand scenarios to better follow the demand variation along the analyzed period as for instance the approaches in $[8,9]$. In this case, the DSR problem aims at identifying the radial topology that is more adequate to the set of demand scenarios under analysis while minimizing the cost of active losses along a planning period.

In this work we propose applying the Specialized Genetic Algorithm of Chu-Beasley (SGACB) described in [10] to solve the DSR problem considering different demand scenarios. The SGACB is a population based metaheuristic in which each individual codes a network topology associated to the connected/disconnected state of each system branch. In this algorithm the population of individuals is submitted to a selection process using a tournament approach. Then, a pair of individuals is subjected to the recombination step in order to generate two sons and the best of them is selected to undergo mutation as well as an improvement process designed to obtain a better value for its fitness function (modelled by the cost of active losses associated to a given topology). In order to evaluate the fitness of each individual (that is, of each topology under analysis) it is run a power flow study for each demand scenario using the approach described in [11] and the corresponding cost of active losses is then calculated considering the duration of each demand scenario. The proposed paper includes results obtained for test systems having 33, 70, 84 and 136 buses and for a real system with 417 buses. The obtained results confirm the robustness and the efficiency of the algorithm, demonstrating the quality of the proposed approach.

\section{Proposed Methodology}

\section{A. Coding the candidate solutions}

In this work we used the scheme detailed in [12] to code the candidate topologies that are identified along the solution algorithm. This scheme uses integer numbers to indicate the branches of the system that are opened/disconnected. If $n_{l}$ represents the number of branches of the system and $n_{b}$ the number of buses, then $L F$ given by (1) is the number of 
fundamental loops of the graph associated to the network under analysis. From Graph Theory, $L F$ gives the minimum number of loops in the graph so that by means of their combinations it is possible to obtain any other loop in the graph. The fundamental loops should then be selected and the branches in each of them should be stored.

$$
L F=n_{l}-n_{b}+1
$$

For illustration purposes, Figure 1 presents a test system having 14 buses and 16 branches. As a result, the associated graph has 3 fundamental loops as illustrated in Figure 1 (Loops 1, 2 and 3) and detailed in (2).

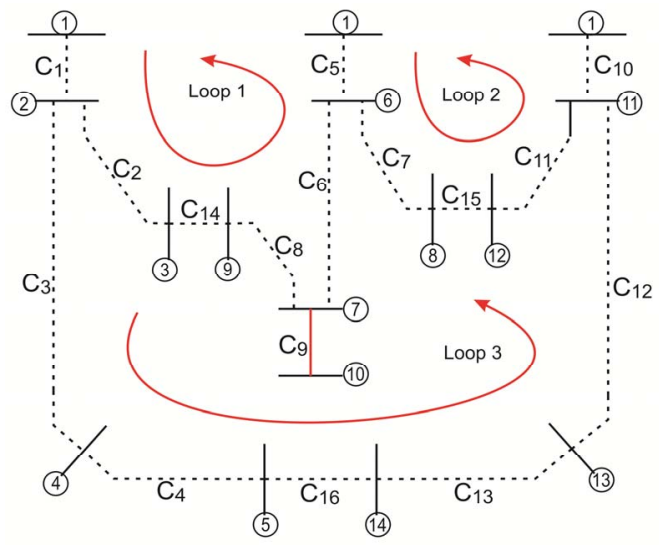

Figure 1. Fundamental loops for the 14 bus test system.

$$
\begin{gathered}
L_{1}=\left[C_{1}, C_{2}, C_{14}, C_{8}, C_{6}, C_{5}\right] \\
L_{2}=\left[C_{5}, C_{7}, C_{15}, C_{11}, C_{10}\right] \\
L_{3}=\left[C_{1}, C_{3}, C_{4}, C_{16}, C_{13}, C_{12}, C_{10}\right]
\end{gathered}
$$

The circuit $\mathrm{C}_{9}$ does not belong to any fundamental loop because it is a terminal circuit. This circuit is not part of the system's graph, and we assume that its state remains fixed, that it is connected during the application of the algorithm so that the demand in node 10 is always supplied.

After identifying the fundamental loops, one branch in each loop is selected to be disconnected so that the set of remaining branches are assumed as connected while generating a radial topology. For instance, if one selects branches $\mathrm{C}_{2}, \mathrm{C}_{15}$ and $\mathrm{C}_{16}$, the vector that codes the associated radial topology is given by (3).

$$
\left[\begin{array}{lll}
2 & 15 & 16
\end{array}\right]
$$

\section{B. SGACB applied to the DSR with different demand levels}

The SGACB algorithm was proposed in [10] and it corresponds to the specialized version of the Chu-Beasley algorithm detailed in [13].

Figure 2 illustrates the flowchart of the SGACB algorithm applied to the solution of the DSR problem for different demand scenarios. The next paragraphs explain the main steps of the developed methodology.

Step I: The initial population is generated using the heuristic described in [12]. This heuristic uses the fundamental loops to randomly generate candidate topologies. Pseudocode 1 describes in more detail the main steps of this heuristic. This strategy makes it possible to generate solutions that are topologically feasible.

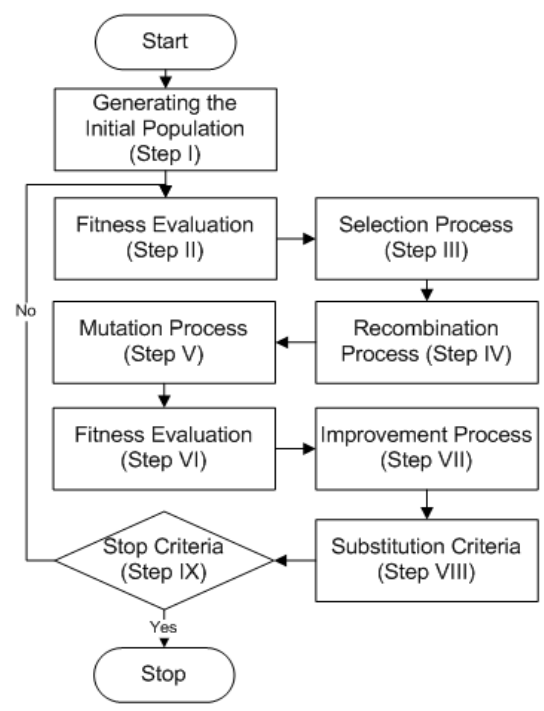

Figure 2. Flowchart of the SGACB.

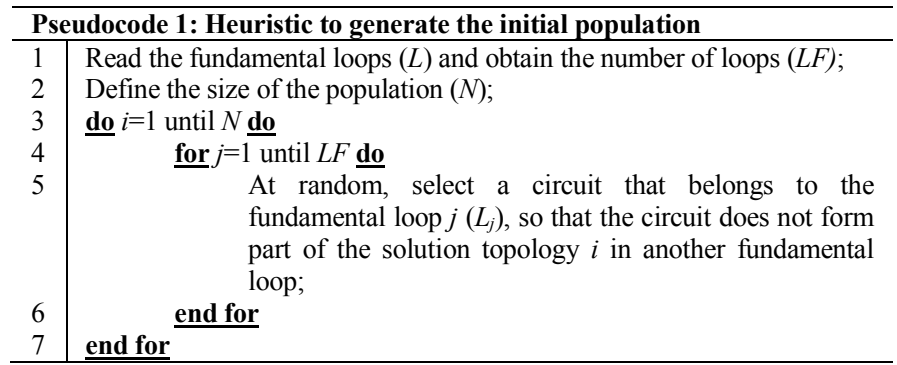

Step II: Evaluate the quality of each individual in the population calculating its fitness. The fitness function represents the cost of energy losses along a full year of operation. In the developed approach we considered three demand scenarios (large, medium and reduced) meaning that a power flow algorithm is run for each of them using [11] in order to calculate the active losses. After obtaining the active losses for each scenario, the cost of the energy losses along a full year is calculated using (4). In this expression, $K$ represents the cost of energy, $T_{1}, T_{2}$ and $T_{3}$ are the durations of each demand scenario and $P_{1}, P_{2}$ and $P_{3}$ are the corresponding active power losses.

$$
f=K *\left[T_{1} * P_{1}+T_{2} * P_{2}+T_{3} * P_{3}\right]
$$

Additionally it is calculated the $U F$ coefficient for each individual in the population measuring its unfeasibility degree in all the demand scenarios to be analyzed. The $U F$ is calculated for all the demand scenarios and it is used in the substitution Step VIII. This parameter is given by the sum of the absolute values of the deviations of nodal voltages regarding the specified ranges plus the sum of the deviations of the branch currents regarding the thermal limits of each branch. If $U F$ is zero, then the associated topology can be implemented from a technical point of view in all demand scenarios;

Step III: Perform the tournament selection process, using pairs of individuals in the population $\left(\mathrm{S}_{1}\right.$ and $\left.\mathrm{S}_{2}\right)$; 
Step IV: Perform the recombination step generating two children individuals from two parents. This process is based in [12] and the main steps of this procedure are detailed in the Pseudocode 2.

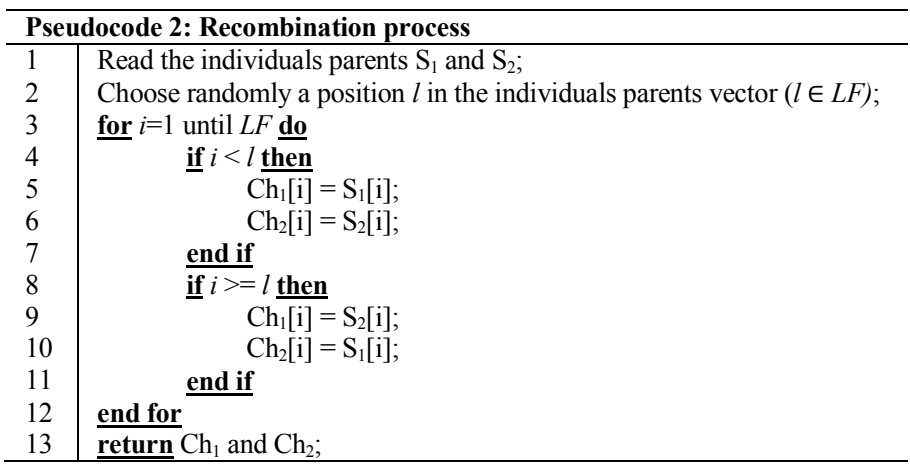

An example of the recombination process is provided in Figure 3. After getting the individuals $\mathrm{S}_{1}$ and $\mathrm{S}_{2}$, it is randomly chosen a position to implement the recombination, that is a crossover point. Then, using this crossover point, the children individuals $\left(\mathrm{Ch}_{1}\right.$ and $\left.\mathrm{Ch}_{2}\right)$ are generated by combining the characteristics of the parents. This recombination strategy ensures that the child individuals are topologically feasible, that is, they remain associated to radial topologies.

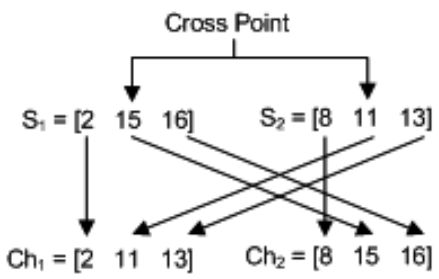

Figure 3. Recombination process.

Step V: Evaluate the fitness function of each child. Eliminate the one having the largest fitness value from the pair generated in Step IV and keep the one having the lowest fitness value;

Step VI: Using the mutation strategy described in [12], perform the mutation step on the child that was selected in Step V. This process is detailed in the Pseudocode 3.

\begin{tabular}{l|l}
\hline \multicolumn{2}{l}{ Pseudocode 3: Mutation process } \\
\hline 1 & $\begin{array}{l}\text { Read the child selected in step V }(\mathrm{Ch}) ; \\
\text { Select randomly a position } l \text { of the child. This position } l \text { represents } \\
\text { a fundamental loop }(l \in L F) ;\end{array}$ \\
3 & $\begin{array}{l}\text { Choose a circuit of the fundamental loop selected that is not } \\
\text { disconnected in another position of the child and use it to replace } \\
\text { the circuit of the position } l ;\end{array}$ \\
4 & $\begin{array}{l}\text { Store the child muted in } \mathrm{Ch} * \\
\text { return }\end{array}$
\end{tabular}

An example for this process is presented in Figure 4.This process involves the random selection of a position in the vector coding the selected child $(\mathrm{Ch})$. This position is associated to a branch included in a fundamental loop. Then a branch should be selected in this loop provided it is not yet taken as connected in another fundamental loop. Then, the randomly selected branch should be exchanged by this new one. Finally, a mutated children $\left(\mathrm{Ch}^{*}\right)$ is obtained.

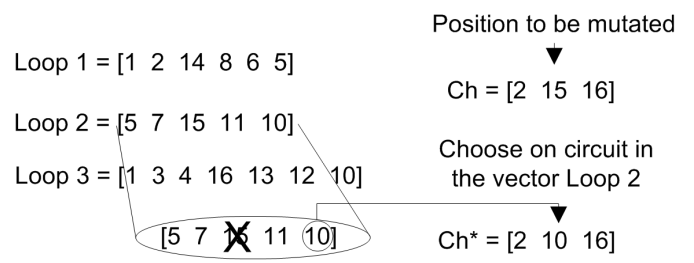

Figure 4. Mutation process.

Step VII: The mutated son should now undergo through an improvement process using the local heuristic search described in [14]. In a brief way, in this process all the branches are analyzed, considering that each of them is associated to a fundamental loop. For each branch, the associated fundamental loop is identified and then in this loop the opened branch in it is taken. This branch is now connected and two new topologies are tested, one of them opening the upstream branch and the other opening the downstream branch. If the fitness function of each of these two new topologies is better than the one of the departing topology, then this new solution is accepted. This procedure is repeated until all the branches in the mutated son are analyzed.

Step VIII: Evaluate if the improved child can substitute any individual in the current population. The criterium to make this substitution is as follows. In the first place, check if this mutated improved child is already in the current population. If it is, then this mutated child is eliminated. If not:

(a) if the mutated child corresponds to a solution that is not feasible (if $U F$ is not zero) then it can only substitute an individual in the population that is also not feasible. The substitution takes place if the degree of unfeasibility $(U F)$ of the mutated child is smaller than the $U F$ value of the unfeasible individual in the current population that has the largest $U F$. If the $U F$ of the mutated child is even larger than the largest $U F$ of the unfeasible individuals of the current population then that mutated child is eliminated;

(b) If the mutated child is feasible (if $U F$ is zero) and there are unfeasible individuals in the current population, then the mutated child replaces the one having the largest $U F$. If no unfeasible individuals exist in the current population, then the mutated child replaces the worse of the individuals in the population provided that its fitness value is smaller. If not, then the mutated child is eliminated;

Step IX: Evaluate convergence. Convergence is obtained if the fitness value of the best individual in the population did not change for a specified number of iterations and the average value of the fitness value of the individuals in the population doesn't change more than a specified percentage along a specified number of iterations. If convergence was obtained, then Stop indicating that a final solution was obtained. If not, increase the iteration counter by 1 . If the maximum number of iterations was reached then Stop indicating that a final solution was not obtained. If not, return to Step II.

\section{RESULTS}

The SGACB algorithm was implemented in Borland $\mathrm{C}++$ $6.0 \AA$ and the tests were conducted in a PC Intel Core 2 Duo $1.9 \mathrm{GHz}$. The tests were performed using test systems with 33, 70,84 and 136 buses and a real system with 417 buses. The 
data of these five test systems can be obtained in [3], [1], [15], [5] and in [16].

Regarding the demand, we considered three scenarios termed as large, medium and reduced. The larger level corresponds to the demand specified for the systems under analysis, while the medium and the reduced scenarios are obtained multiplying the larger demand values by 0.80 and by 0.50 . Finally, the simulations considered a full operation year and the durations of the larger, medium and reduced demand levels were set at 1000, 6760 and 1000 hours per year. The cost of energy losses was set at $0.0468 € / \mathrm{kWh}$.

\section{A. Test systems with 33, 70, 84 and 136 Buses}

The test systems having 33, 70, 84 and 136 buses have nominal voltage of $12.66 \mathrm{kV}, 12.66 \mathrm{kV}, 11.40 \mathrm{kV}$ and 13.80 $\mathrm{kV}$. The tests were performed using the parameters presented in Table I in which $N$ is the number of individuals in the population and itera is the maximum number of iterations.

TABLE I. Parameters used for the test systems in the SGACB.

\begin{tabular}{c|c|c}
\hline \multirow{2}{*}{ Parameters } & \multicolumn{2}{|c}{ Distribution system } \\
\cline { 2 - 3 } & 33,70 and 84 buses & 136 buses \\
\hline$N$ & 30 & 50 \\
\hline itera & 30 & 100 \\
\hline
\end{tabular}

Table II presents the energy cost for a full operation year for the initial topology and for the final one provided by the SGACB each of the four test systems. Given that the SGACB is a population based algorithm, the final solution corresponds to the solution in the final population that has the lowest value of the fitness function, that is, the most reduced energy losses along the entire simulated year.

Table III details the initial and the final identified topologies for each of the four tested systems. These final four topologies are feasible from a technical point of view, that is, the final value of the $U F$ coefficient is zero for all of them.

TABLE II. Cost of energy losses $(€)$ for test systems.

\begin{tabular}{c|c|c|c}
\hline System & $\begin{array}{c}\text { Cost of energy losses } \\
\text { Initial topology }(€)\end{array}$ & $\begin{array}{c}\text { Cost of energy losses } \\
\text { Final topology }(€)\end{array}$ & $\begin{array}{c}\text { Reduction } \\
(\%)\end{array}$ \\
\hline 33 & 51488.28 & 35798.53 & 30.47 \\
\hline 70 & 5416.61 & 2445.31 & 54.85 \\
\hline 84 & 136610.07 & 121040.01 & 11.39 \\
\hline 136 & 82417.68 & 72372.93 & 12.18 \\
\hline
\end{tabular}

TABLE III. Initial and final topologies for test systems.

\begin{tabular}{|c|c|c|}
\hline System & Topology & Disconnected branches \\
\hline \multirow{2}{*}{$\begin{array}{c}33 \\
\text { buses }\end{array}$} & Initial & $33-34-35-36-37$ \\
\hline & Final & $7-9-14-32-37$ \\
\hline \multirow{2}{*}{$\begin{array}{c}70 \\
\text { buses }\end{array}$} & Initial & $70-71-72-73-74$ \\
\hline & Final & $15-56-62-70-71$ \\
\hline \multirow{2}{*}{$\begin{array}{c}84 \\
\text { buses }\end{array}$} & Initial & 84-85-86-87-88-89-90-91-92-93-94-95-96 \\
\hline & Final & $7-13-34-39-42-55-62-72-83-86-89-90-92$ \\
\hline \multirow{2}{*}{$\begin{array}{c}136 \\
\text { buses }\end{array}$} & Initial & $\begin{array}{l}\text { 136-137-138-139-140-141-142-143-144-145-146- } \\
147-148-149-150-151-152-153-153-154-155-156\end{array}$ \\
\hline & Final & $\begin{array}{l}\text { 7-35-51-90-96-106-118-126-135-137-138-141-142- } \\
144-145-146-147-148-150-151-155\end{array}$ \\
\hline
\end{tabular}

The computation time taken to identify the final topologies presented in the Table III, is provided in Table IV.

Figure 5 shows the voltage profile of the 33 bus system, before the reconfiguration process (a) and after the reconfiguration process (b). It can be observed that after the reconfiguration the voltage levels are improved and all nodal voltages comply with the specified limits.

TABLE IV. Computation time for test systems.

\begin{tabular}{c|c}
\hline System & $\begin{array}{c}\text { Computation time } \\
\text { (seconds) }\end{array}$ \\
\hline 33 & 0.109 \\
\hline 70 & 0.156 \\
\hline 84 & 1.563 \\
\hline 136 & 8.281 \\
\hline
\end{tabular}
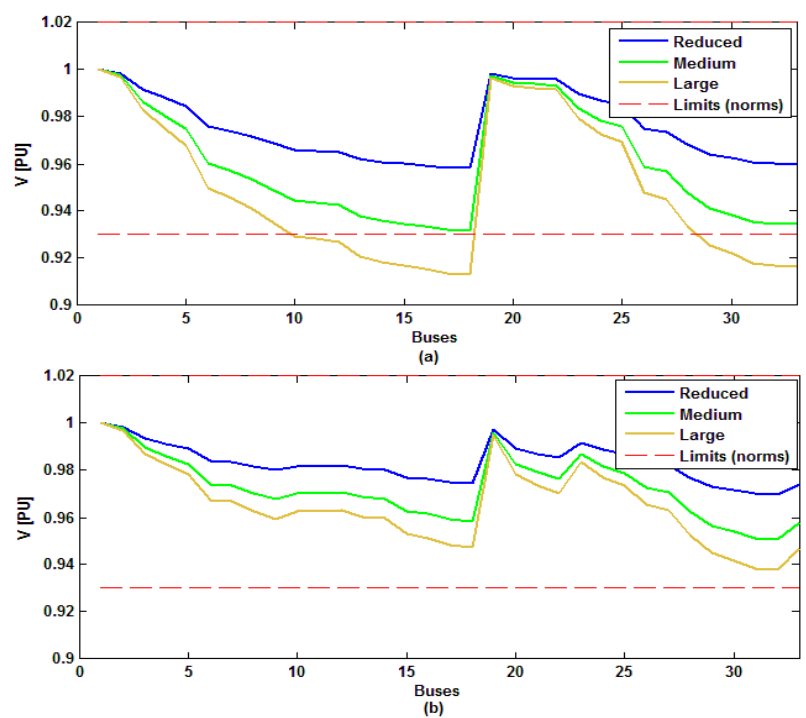

Figure 5. Voltage profile of the 33 buses system.

Figure 6 shows the voltage profile for the 84 bus system before the reconfiguration process (a) and after the reconfiguration process (b). Once again, after the reconfiguration the voltage levels are improved and all nodal voltages are within their limits.
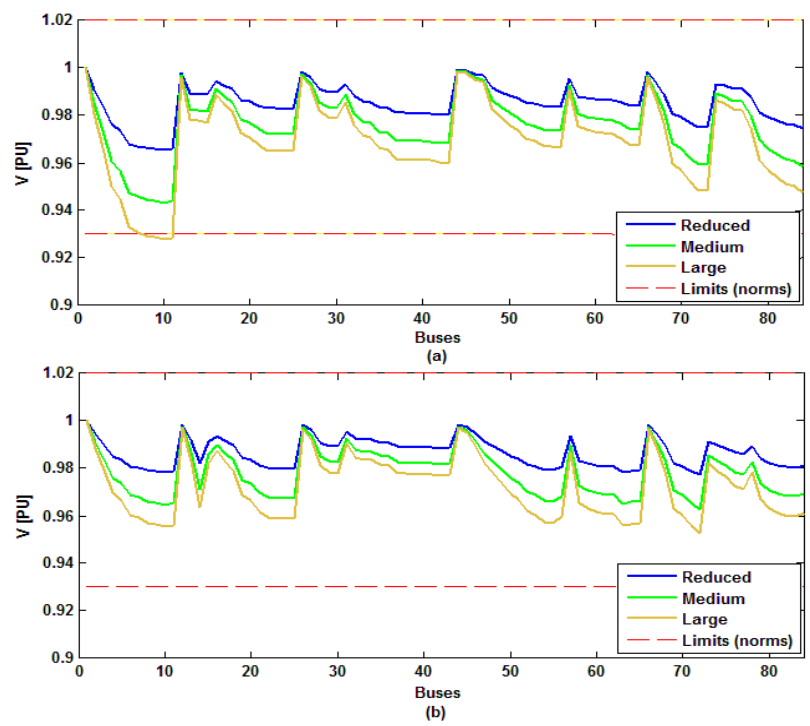

Figure 6. Voltage profile of the 84 buses system.

It is noteworthy that the final topologies obtained for the DSR problem considering several demands, for all test systems, were the same as the ones identified considering a single demand, as presented in [7]. 


\section{B. Real systems with 417 Buses}

The real system with 417 buses has a nominal voltage of $13.8 \mathrm{kV}$ and it is based on a real distribution network [16]. The parameters in Table $\mathrm{V}$ were used in the developed tests.

Table VI presents the energy cost for a full operation year for the initial topology and for the final one for the real systems with 417 buses.

Table VII details the initial and the final identified topologies for the real systems with 417 buses. The final identifies topology is feasible from a technical point of view, which means that the UF coefficient is zero.

The computation time taken to identify the final topology for this system is presented in the Table VIII.

TABLE V. Parameters used for the 417 bus systems in the SGACB.

\begin{tabular}{c|c}
\hline \multirow{2}{*}{ Parameters } & Distribution system \\
\cline { 2 - 2 } & 417 buses \\
\hline$N$ & 80 \\
\hline itera & 200 \\
\hline
\end{tabular}

TABLE VI. Cost of energy losses ( $€$ ) for the 417 bus system.

\begin{tabular}{c|c|c|c}
\hline System & $\begin{array}{c}\text { Cost of energy losses } \\
\text { Initial topology }(€)\end{array}$ & $\begin{array}{c}\text { Cost of energy losses } \\
\text { Final topology }(€)\end{array}$ & $\begin{array}{c}\text { Reduction } \\
(\%)\end{array}$ \\
\hline 417 & 181961.10 & 149948.30 & 17.59 \\
\hline
\end{tabular}

TABLE VII. Initial and final topologies for the 417 bus system.

\begin{tabular}{|c|c|c|}
\hline System & Topology & Disconnected branches \\
\hline \multirow{2}{*}{$\begin{array}{c}417 \\
\text { buses }\end{array}$} & Initial & $\begin{array}{l}1-5-15-16-26-31-53-54-55-75-82-94-96-97-106- \\
107-119-136-138-154-155-156-168-169-177-179- \\
194-195-201-207-211-214-219-241-256-258-282- \\
297-302-314-321-354-359-362-364-385-388-395- \\
396-404-407-423-424-426-431-436-445-446-449\end{array}$ \\
\hline & Final & $\begin{array}{l}\text { 5- 13- 15- 16- 21- 26- 31-54- 57- 59-60-73- 86- 87- } \\
\text { 94- 96- 97-111-115- 136-142-149-150-155-156-158- } \\
\text { 163- 168-169-178-179-191-195-199-214- 221-254- } \\
256-266-282-317-322-325-358-362-369-392-395- \\
403-404-416-423-426-431-436-437-446-449-466\end{array}$ \\
\hline
\end{tabular}

TABLE VIII. Computation time for the 417 bus system.

\begin{tabular}{c|c}
\hline System & $\begin{array}{c}\text { Computation time } \\
\text { (seconds) }\end{array}$ \\
\hline 417 & 22.358 \\
\hline
\end{tabular}

The final topology obtained for the DSR problem considering several demands, for the real systems with 417 buses was the same one that was obtained for the DSR problem considering a single demand, as presented in [7].

\section{CONCLUSIONS}

This work reports the application of the SGACB algorithm to the DSR problem considering different demand scenarios. The objective of the problem is the identification of the topology of the network that minimizes the active losses along a full operation year while enforcing a several operation constraints, for instance related with nodal voltage and branch current limits. The SGACB is easily applied to this problem and with a single run it provides feasible solutions from a technical point of view for all the demand scenarios that were analyzed. The algorithm proved to be robust, fast and efficient even considering the 417 bus real distribution network. Future publications will report improvements of this model namely to consider a maximum number of topology changes regarding an initial one to be specified by the operator. This will contribute to turn this approach more realistic and increase its applicability in distribution control centers.

\section{ACKNOWLEDGEMENT}

The first author wishes to thank INESC TEC, Porto, Portugal, $\mathrm{CNPq} /$ Brazil and CAPES-PDSE/Brazil (proc. $\mathrm{N}^{\circ}$ BEX 3660/14-1) for supporting this research.

The research leading to this work is being carried out as part of the evolvDSO project (Development of methodologies and tools for new and evolving DSO roles for efficient DRES integration in distribution networks) - www.evolvdso.eu. This project is funded by the European Commission under the $7^{\text {th }}$ framework program (FP7) under grant agreement 608732.

\section{REFERENCES}

[1] M. A. N. Guimarães, J. F. C. Lorenzetti, C. A. Castro, "Reconfiguration of distribution systems for voltage stability margin enhancement using tabu search", in Proc. PowerCon, vol. 2, pp. 1556-1561, 2004.

[2] A. Merlin, H. Back, "Search for a minimal-loss operating spinning tree configuration in an urban power distribution system", in Proc. 5th Power System Computation Conference, Cambridge, pp. 1-18, 1975.

[3] M. E. Baran, F. F. Wu, "Network reconfiguration in distribution systems for loss reduction and load balancing", IEEE Trans. on Power Delivery, vol. 4, n. 2, pp. 1401-1407, 1989.

[4] K. Nara, A. Shiose, M. Kitagawa, T. Ishihara, "Implementation of genetic algorithm for distribution systems loss minimum reconfiguration”, IEEE Transactions on Power Systems, vol. 7, n. 3, pp. 1044-1051, 1992.

[5] D. Zhang, Z. Fu, L. Zhang, "An improved TS algorithm for lossminimum reconfiguration in large-scale distribution systems," Electric Power Systems Research, vol. 77, no. 5-6, pp. 685-694, 2007.

[6] E. Carpaneto, G. Chicco, "Distribution system minimum loss reconfiguration in the hyper-cube ant colony optimization framework", Electric Power Systems Research, vol. 78, pp. 2037-2045, 2008.

[7] S. S. F. Souza, R. Romero, J. F. Franco, "Artificial immune networks Copt-aiNet and Opt-aiNet applied to the reconfiguration problem of radial electrical distribution systems," Electric Power Systems Research, vol. 119, pp. 304-312, 2015.

[8] L. Queiroz, C. Lyra, "Adaptive hybrid genetic algorithm for technical loss reduction in distribution networks under variable demands", IEEE Trans. on Power Systems, vol. 24, no. 1, pp. 445-453, 2009.

[9] T. Inoue, K Takano, T. Watanabe, J. Kawahara, "Distribution loss minimization with Guaranteed error bound", IEEE Trans. on Smart Grid, vol. 5, no. 1, pp. 102-111, 2014.

[10] I. J. Silva, M. J. Rider, R. Romero, A. V. Garcia, C. A. Murari, "Transmission network expansion planning with security constraints," in IEE Proc. G.T.\&D., vol. 152, no. 6, pp. 828-836, Nov. 2005.

[11] D. Shirmohammadi, H. W. Hong, A. Semlyen, G. X. Luo, "A compensation based power flow method for weakly meshed distribution and transmission networks," IEEE Trans. on Power Systems, vol. 3, no. 2, pp. 753-762, 1988.

[12] J. Mendoza, R. Lopez, D. Morales, E. Lopez, P. Dessante, R. Moraga, "Minimal loss reconfiguration using genetic algorithms with restricted population and addressed operators: Real applications", IEEE Trans. on Power Systems, vol. 21, no. 2, pp. 948-954, 2006.

[13] P. Chu, J. E. Beasley, "A genetic algorithm for the generalized assignment problem", Computers and Operations Research, vol. 24, no. 1, pp. 17-23, 1997.

[14] E. M. Carreño, R. Romero, A. P. Feltrin, "An efficient codification to solve distribution network reconfiguration for loss reduction problem", IEEE Trans. on Power Systems, vol. 23, no. 4, pp. 1542-1551, 2008.

[15] J. P. Chiou, C. F. Chang, C. T. Su, "Variable scaling hybrid differential evolution for solving network reconfiguration of distribution systems", IEEE Trans. on Power Systems, vol. 20, no. 2, pp. 668-674, 2005.

[16] J. L. Bernal-Agustin, "Application of genetic algorithms to the optimal design of power distribution systems", 1998. $346 \mathrm{f}$. Tesis, University of Zaragoza, Zaragoza, 1998. 